\title{
Pulmonary Mucormycosis in a Child Diagnostic by CT and the Importance of the Reverse Halo Signal in Differential Diagnosis
}

\author{
Lima CMA01* ${ }^{*}$ Maymone W1, Porto MTC1, Penna CRR ${ }^{1}$, David SG², \\ Arantes $\mathrm{AA}^{3}$ and Fazecas $\mathrm{T}^{4}$ \\ ${ }^{1}$ Pediatrics Radiologists at Jesus Municipal Hospital, Brazil \\ ${ }^{2}$ Head of the Pediatric Pulmonology Service of Jesus Municipal Hospital, Brazil \\ ${ }^{3}$ Pediatric Pneumologist and Pediatric Endoscopist of Jesus Municipal Hospital, Brazil \\ ${ }^{4}$ Head of the Pediatric Radiology Service of Jesus Municipal Hospital, Brazil
}

\section{Image Article \\ Volume 3 Issue 2}

Received Date: February 14, 2019

Published Date: March 18, 2019

*Corresponding author: Cláudio Márcio Amaral de Oliveira Lima, MD, Pediatric Radiology Service of Jesus Municipal Hospital, Rio de Janeiro, Brazil, 717 December 8th St, Rio de Janeiro, RJ, Brazil 20550-200, Tel: +55(21)9987184089; Email: cmaolima@gmail.com

\section{Abstract}

Mucormycosis or zygomycosis is an uncommon opportunistic fungal infection caused by fungi of the order Mucorales in the class Zygomycetes. It's far less common than other opportunistic fungal infections, such as Aspergillus and Candida infections, although the mortality rate is much higher. The three most common primary clinical manifestations of mucormycosis are rhinocerebral, pulmonary, and cutaneous infections. The clinical manifestations include fever, cough, hemoptysis, and dyspnea. Diabetes mellitus is a common predisposing factor for mucormycosis. Pulmonary mucormycosis is the most frequent cause of the reversed halo sign in patients with hematologic diseases and those who have undergone bone marrow transplant two other pulmonary diseases.

Keywords: Pulmonary; Mucormycosis; CT; Reverse Halo Signal; Child

Abbreviations: CT: Computed Tomography; RHS: Reverse Halo Sign; PM: Pulmonary Mucormycosis; OP: Organizing Pneumonia.

\section{Introduction}

Zygomycosis are parasitic diseases caused by Mucorales species, are those of the genera Rhizopus, Lichtheimia and Mucor. It's far less common than other opportunistic fungal infections, such as Aspergillus and
Candida infections, although the mortality rate is much higher. The three most common primary clinical manifestations of mucormycosis are rhinocerebral, pulmonary, and cutaneous infections [1,2]. Immunocompromised hosts and diabetes are important risk factors. In contrast to the CT halo sign, the reverse halo sign (RHS) is seen in association with pulmonary mucormycosis (PM) [3]. We present a case of PM with the typical RHS and we discuss the importance of this signal and its most important differential diagnoses. 


\section{Clinical Radiology \& Imaging Journal}

\section{Case Report}

Female 11 years, diagnosis of Type 1 Diabetes at 8 years of age. Beginning two months of dry cough without dyspnea, fever, tiredness or weight loss. She performed chest X-ray showing homogeneous consolidation in the lower left lobe (Figure 1A).

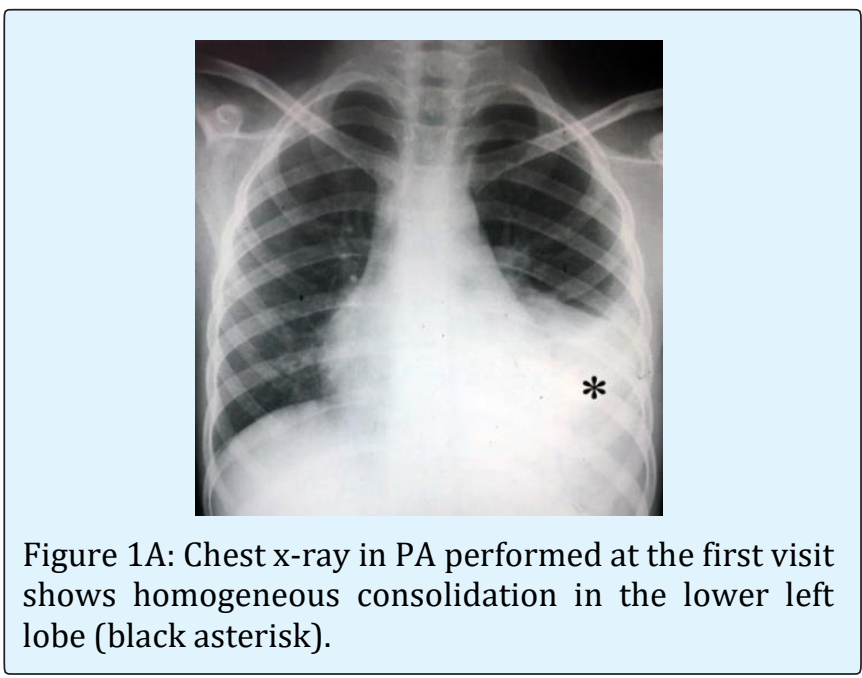

Antibiotic therapy prescribed. It returns after approximately 45 days with clinical persistence. New chest $\mathrm{x}$-ray shows that consolidation is maintained, but now shows areas of cavitation (Figure 1B). Glycemia of $464 \mathrm{mg} / \mathrm{dl}$. The physical examination is in good general condition and reactive, without respiratory effort. Vesicular murmur reduced in the middle third of the left hemitorax. SO2 99\% and C-Reactive Protein $=7.3 \mathrm{mg} / \mathrm{dl}$. CT scan of the chest shows consolidation with air bronchograms and an atelectasic component in the left lower lobe with rounded formation of thick walls, with an area of cystic-necrotic degeneration in the interior, without contrast enhancement.

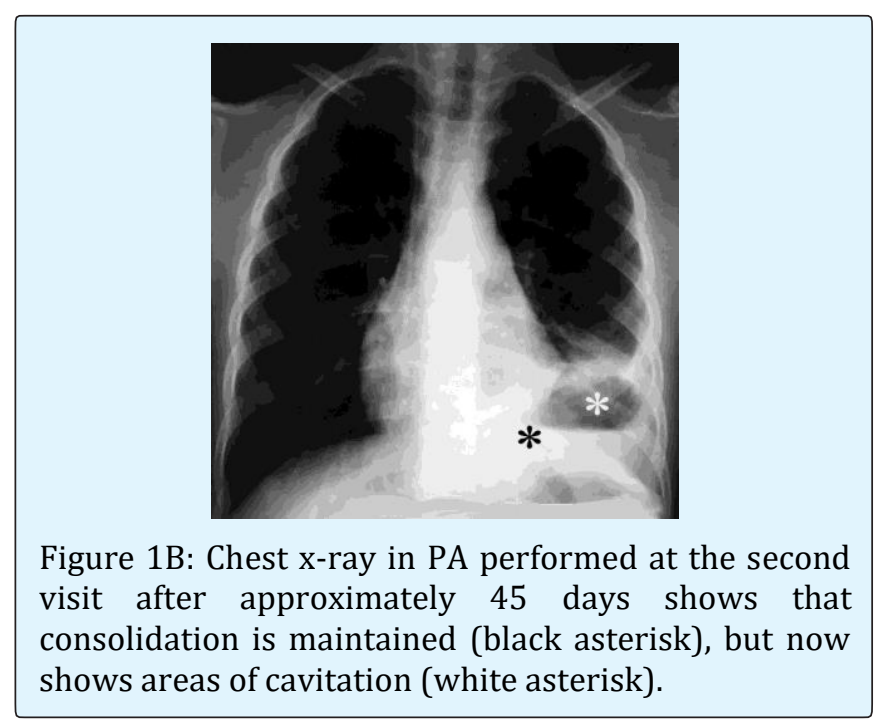

The classical RHS is a focal rounded area of groundglass opacity surrounded by more ou less complete ring of consolidation. The described set determines cranial traction of the diaphragmatic hemicule on the same side (Figures 2A-B). Bronchoscopy was performed with bronchoalveolar lavage and collection of material for culture. Results were negative for tuberculosis and positive for mucormycosis. Initiation of treatment with amphotericin B. presented two episodes of hemoptysis during hospitalization. Control CT performed approximately 40 days later showed that there was a slight reduction in size of the lesion (Figures 3A-B).
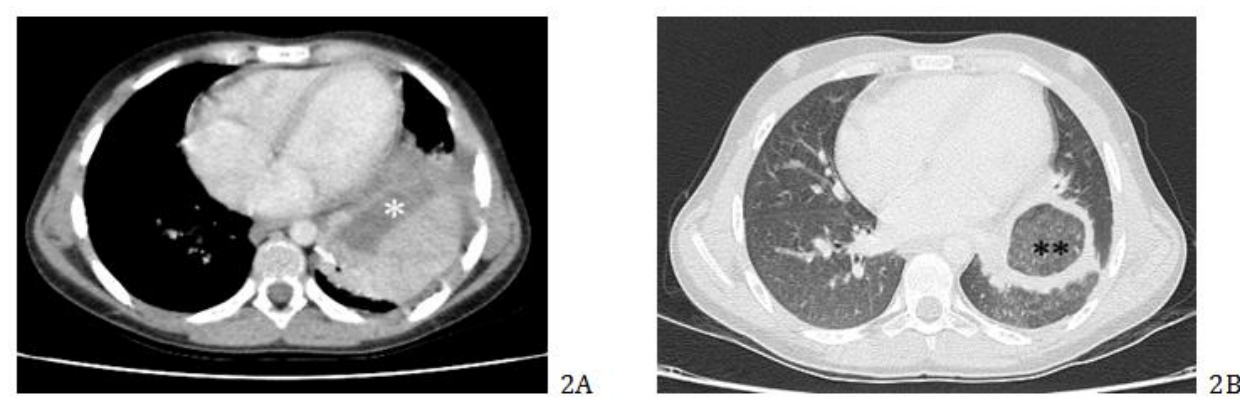

Figures 2(A-B): First CT of the chest after intravenous contrast administration. Axial image with mediastinal window (A) and pulmonary window (B). In A, consolidation (white asterisk) with air bronchograms (white arrow) and an atelectasic component in the left lower lobe with rounded formation of thick walls, with an area of cystic-necrotic degeneration in the interior, without contrast enhancement. In B, the classical reverse halo sign is a focal rounded area of ground-glass opacity surrounded by more ou less complete ring of consolidation (double black asterisk). 


\section{Clinical Radiology \& Imaging Journal}
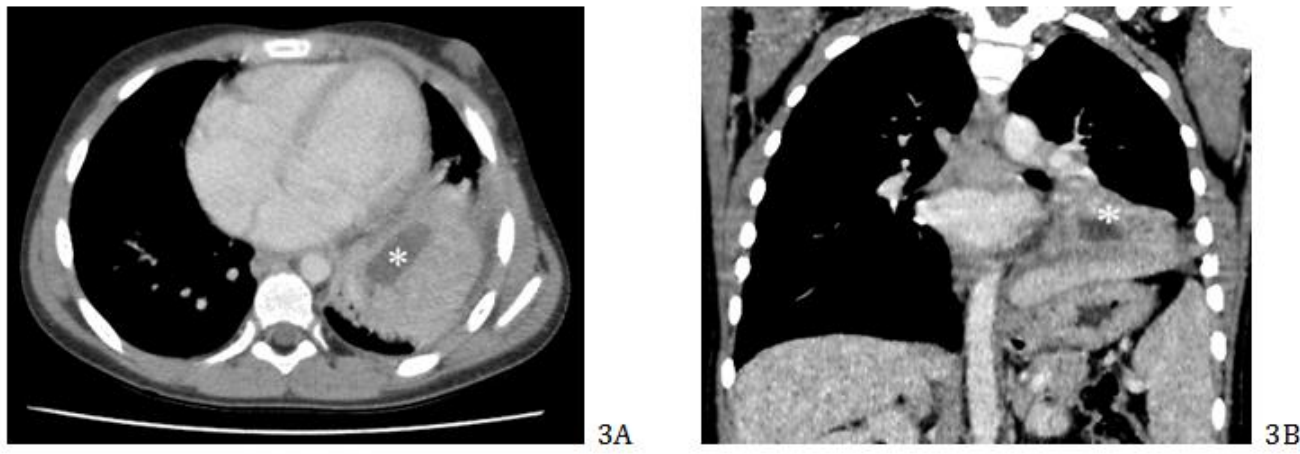

Figures 3A-B: Control CT of the chest after intravenous contrast administration performed approximately 40 days later. Axial image (A) and coronal image (B) with mediastinal window shows a slight reduction in size of the consolidation but still with central area of necrosis (white asterisk).

On the 10th day of hospital stay, there was alteration in renal scaling levels, when the antifungal was changed to lysosomal amphotericin B. It evolved with slight clinical improvement. A further CT scan is performed after approximately 35 days, which shows reduction of the consolidation with an atelectasic component in the left lower lobe without evidence of necrosis area inside (Figures 4A-D).
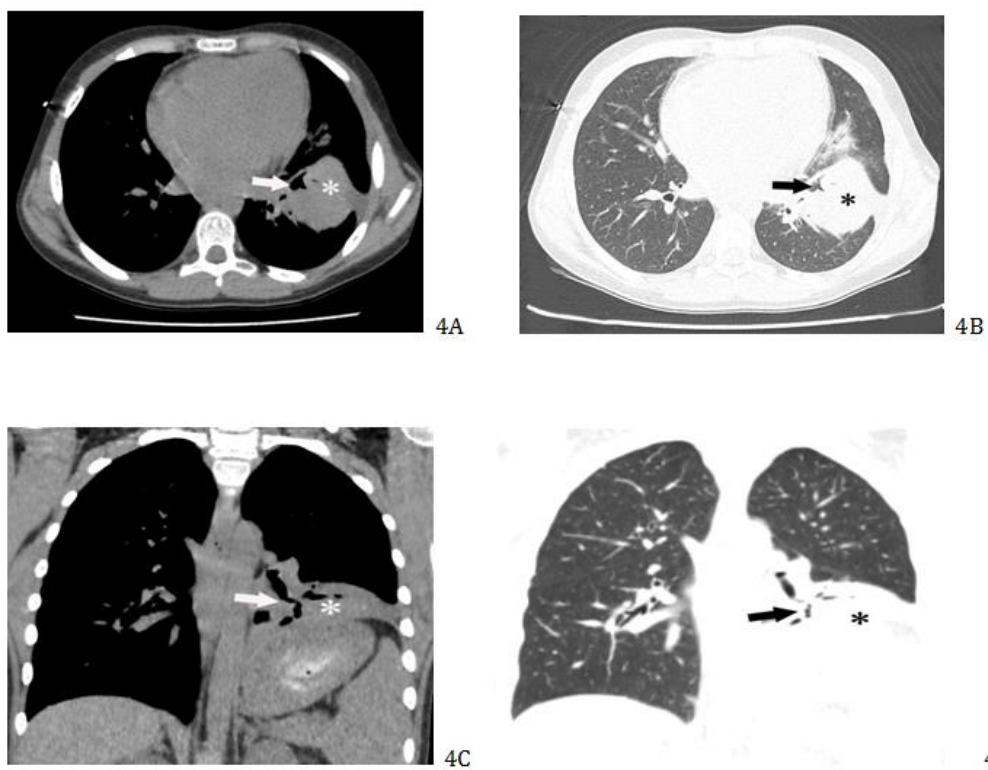

$4 \mathrm{D}$

Figures 4A-D: CT of the chest performed after approximately 35 days. Axial image with mediastinal window (A) and pulmonary window (B) and coronal image with mediastinal window (C) and pulmonary window (D) shows reduction of the consolidation (white asterisk in A and C, black asterisk in B and D) with air bronchograms (white arrow in A and C, black arrow in B and D) and atelectasic component in the left lower lobe without evidence of necrosis area inside.

She remains hospitalized with significant clinical and radiological improvement. New bronchoscopy with bronchoalveolar lavage was negative for fungi. The last chest $\mathrm{x}$-ray performed after approximately 20 days shows that there is no cavitation as well as there is improvement 


\section{Clinical Radiology \& Imaging Journal}

in the size of the consolidation (Figure 5). Scheduled for hospital discharge in 7 days.

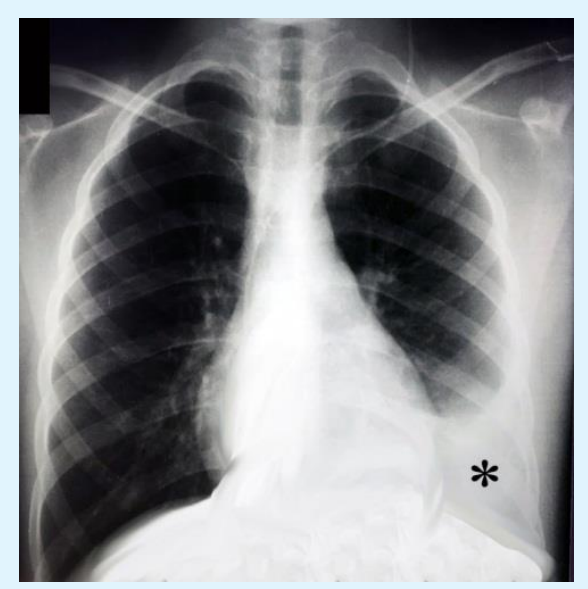

Figure 5: The last chest x-ray in PA performed after approximately 20 days shows that there is no cavitation as well as there is improvement in the size of the consolidation (black asterisk). Scheduled for hospital discharge in 7 days.

\section{Discussion}

Mucormycosis or zygomycosis is an uncommon opportunistic fungal infection caused by fungi of the order Mucorales in the class Zygomycetes. It's far less common than other opportunistic fungal infections, such as Aspergillus and Candida infections, although the mortality rate is much higher. The three most common primary clinical manifestations of mucormycosis are rhinocerebral, pulmonary, and cutaneous infections [1-3].

Mucormycosis was 1st described by Paulltaf in 1885, has emerged as the third most common invasive mycosis in order of importance after candidiasis and aspergillosis in patients with hematological and allogeneic stem cell transplantation $[3,4]$. Various risk factors have been associated with mucormycosis like malignant hematological disease with or without stem cell transplantation, prolonged and severe neutropenia, poorly controlled diabetes mellitus, iron overload, prolonged use of steroids, intravenous drug use, graftversus-host disease, neonatal prematurity, prolonged use of antifungal (voriconazole) and children who are critically ill or bear placed catheters or prostheses $[1,5]$. The increased release of iron from ferritin which occurs because of acidosis caused enhanced fungal and hyphae growth [5]. Fungal growth and proliferation is promoted by certain metabolic states like hyperglycemia and acidosis. Diabetes mellitus is a most common predisposing factor for mucormycosis in adults, present in $36-88 \%$ of cases. Bhat A [6] in a retrospective study that included all hospitalized patients with confirmed mucormycosis disease within a year $(n=7)$ reported that the main risk factor in all of these cases was uncontrolled diabetes mellitus (71.4\%). In children, diabetes is present in only $15 \%$ of patients with mucormycosis but are associated with significant morbidity and death $[1,2]$. Mucormycosis has shown an equal sex distribution. But, there is slightly male predominance and the ratio of male: female is 1.3:1 [6]. In our case, the patient was female and had type I diabetes.

The clinical manifestations of the PM include fever, cough, hemoptysis, and dyspnea. Further complicating early diagnosis is the frequent co-existence of other infections with rates as high as 39\% [3]. Bhat A [6] reports that hemoptysis was the initial presentation in the majority of cases of PM. In our case the initial presentation was dry cough but the patient had two episodes of hemoptysis during hospitalization. Three routes of infections are inhalation, ingestion and traumatic inoculation. Sporangiospores are the infective forms while angioinvasive hyphal forms are responsible for tissue invasion, infarction and dissemination. The ability to scavenge of free iron from the host is essential for growth and pathogenesis. Based on its clinical presentation and anatomic site involvement, invasive mucormycosis is classified into: (1) Rhinocerebral, (2) Pulmonary, (3) Cutaneous, (4) Gastrointestinal, (5) Disseminated, and (6) Uncommon rare forms, such as endocarditis, peritonitis, and renal infection. Rhinocerebral mucormycosis is the most common form [1].

The radiologic manifestations of PM include consolidation, cavitation, abscess formation, nodules, and masses. Pulmonary lesions are usually unifocal and affect the upper lobe. As with other angioinvasive fungal infections, the air-crescent or halo sign can be seen [7]. The CT halo sign is also well described in association with Candida, $C$ immitis, and $C$ neoformans infections. In contrast to the CT halo sign, the RHS-central ground-glass opacity surrounded by consolidation-is seen in association with PM [3]. PM is the most frequent cause of the RHS in patients with hematologic diseases and those who have undergone bone marrow transplant two other pulmonary diseases-organizing pneumonia (OP) and tuberculosis-also need to be considered as important causes of this sign in this population. OP has been reported in patients with hematologic malignancies, in whom it is frequently related to infection, radiation, and 


\section{Clinical Radiology \& Imaging Journal}

chemotherapy. Tuberculosis is not rare among patients with hematologic malignancies in countries with a high incidence of this infection, and it causes significant morbidity and mortality, especially in patients with acute leukemia. Some morphologic characteristics of the RHS aid the differential diagnosis. The presence of nodular walls or nodules inside the RHS strongly favors a diagnosis of active pulmonary tuberculosis, rather than OP. The presence of reticulation inside the RHS, with an outer consolidation rim measuring greater than $1 \mathrm{~cm}$ in thickness, in association with pleural effusion, strongly suggests the diagnosis of PM, rather than OP. The RHS caused by OP does not present these tomographic characteristics [8]. The consolidation caused by PM has a propensity to cavitate. Pulmonary infections due to the fungus-like Actinomyces and Nocardia species also typically cause consolidation that frequently cavitates and involves the pleura and chest wall. This process is known as empyema necessitans [3].

\section{Conclusion}

Mucormycosis is a rare opportunistic fungal infection with rapidly progressive and fulminant course with often fatal outcome. Immunocompromised hosts and diabetes are important risk factors. The role of diagnostic imaging in children is multifactorial, including initially detecting it, evaluating for dissemination of infection beyond the primary site of disease, monitoring the response to antifungal therapy, and assessing for potential relapse.

The RHS can be encountered in a variety of pulmonary disorders, both infectious and noninfectious. The two most commonly associated diseases are the organizing pneumonias and invasive fungal pneumonias (especially mucormycosis). As the treatment approach for these two diseases is diametrically opposite, we recommend obtaining a tissue diagnosis whenever feasible. The clinical presentation, immune status of the patient, and associated radiologic findings can help in narrowing down the differential diagnosis. In an immunocompromised host, antifungal therapy for mucormycosis should be initiated pending further investigations.

\section{References}

1. Kumar S, Kataria SP, Singh G, Parmar P, Yada H, et al. (2018) Invasive gastrointestinal mucormycosis: a rare presentation. EJPMR 5(11): 368-371.

2. Hammer MM, Madan R, Hatabu H (2018) Pulmonary Mucormycosis: Radiologic Features at Presentation and Over Time. AJR Am J Roentgenol 210(4): 742747.

3. Orlowski HLP, McWilliams S, Mellnick VM, Bhalla V, Lubner MG, et al. (2017) Imaging Spectrum of Invasive Fungal and Fungal-like Infections. Radio Graphics 37(4): 1119-1134.

4. Alqhamdi S, Idress B, Alharbi A, Aljurais N (2019) Case Report: Disseminated pulmonary mucormycosis involving spleen in diabetic patient with aggressive surgical approach. Internal J Surg Case Rep 54: 42-46.

5. Thomas S, Pawar B, Fernandes D, Nayar S, George P, et al. (2018) An Unusual Case of Pulmonary Mucormycosis. Transplant Proc 50(10): 3943-3945.

6. Bhat A (2018) Mucormycosis: the clinical spectrum. Int J Adv Med 5(6): 1364-1368.

7. Katragkou A, Fisher BT, Groll AH, Roilides E, Walsh TJ (2017) Diagnostic Imaging and Invasive Fungal Diseases in Children. J Pediatric Infect Dis Soc 6(1): 22-31.

8. Marchiori E, Pereira ML, Zanetti G (2018) The Importance of the Reversed Halo Sign in the Diagnosis of Pulmonary Mucormycosis. AJR Am J Roentgenol 211(2): 137. 